This is an electronic reprint of the original article. This reprint may differ from the original in pagination and typographic detail.

Author(s): Tanskanen, Minna; Atalay, Mustafa; Uusitalo, Arja

Title: $\quad$ Altered oxidative stress in overtrained athletes

Year: $\quad 2010$

Version:

Please cite the original version:

Tanskanen, M., Atalay, M., \& Uusitalo, A. (2010). Altered oxidative stress in overtrained athletes. J Sports Sci, 28(3), 309-317.

https://doi.org/10.1080/02640410903473844

All material supplied via JYX is protected by copyright and other intellectual property rights, and duplication or sale of all or part of any of the repository collections is not permitted, except that material may be duplicated by you for your research use or educational purposes in electronic or print form. You must obtain permission for any other use. Electronic or print copies may not be offered, whether for sale or otherwise to anyone who is not an authorised user. 


\section{Altered Oxidative Stress in Overtrained Athletes}

Minna M. Tanskanen ${ }^{\mathrm{a}}$, Mustafa Atalay ${ }^{\mathrm{b}}$, Arja L. Uusitalo ${ }^{\mathrm{c}, \mathrm{d}, \mathrm{e}}$

${ }^{a}$ Department of Biology of Physical Activity, University of Jyväskylä, Finland

${ }^{\mathrm{b}}$ Institute of Biomedicine, Physiology, University of Kuopio, Finland

${ }^{c}$ Department of Clinical Physiology and Nuclear Medicine, Kuopio University Hospital, Finland

${ }^{\mathrm{d}}$ Institute of Clinical Medicine, Clinical Physiology, University of Kuopio, Finland

e Department of Clinical Physiology and Nuclear Medicine, Helsinki University Hospital, Finland

Corresponding author:

Minna Tanskanen

University of Jyväskylä

Department of Biology of Physical Activity

Kidekuja 2, Snowpolis

88610 VUOKATTI

FINLAND

e-mail: minna.m.tanskanen@jyu.fi

Tel. +358 505948564, Fax +358 86178641

Running title: Oxidative Stress and Overtraining State

Key words: ORAC, Protein Carbonyls, MDA, Nitrotyrosine, Exercise 


\section{Abstract}

The purpose of the present study was to evaluate the association between oxidative stress and overtraining syndrome. Indicators of oxidative stress (plasma protein carbonyls, nitrotyrosine and malondialdehyde and antioxidant status (oxygen radical absorbance capacity [ORAC]) were measured in severely overtrained (two women, five men) and control athletes (five women, five men). Samples were collected from both groups at baseline (i.e. in the overtraining state of overtrained athletes) and after six months of recovery, both at rest and immediately after an exercise test to volitional exhaustion. At baseline, overtrained athletes had higher plasma protein carbonyls at rest than controls (mean difference $0.03 \mathrm{nmol} \cdot \mathrm{mg}^{-1}, 95 \%$ CI $0.01-0.05$ $\mathrm{nmol} \cdot \mathrm{mg}^{-1}, \mathrm{P}=0.003, \mathrm{ES}=0.40$ ). At baseline and following recovery exercise to exhaustion leads to an increase in ORAC and malondialdehyde $(\mathrm{P}=0.001-0.006)$ in the controls but not in the overtrained athletes. Furthermore, at baseline, only overtrained athletes showed negative correlations between ORAC at rest and protein carbonyls after exhaustive exercise $(\mathrm{r}=-0.98, \mathrm{P}=0.0001)$. These results suggest that increased oxidative stress has a role in the pathophysiology of overtraining syndrome. The attenuated responses of oxidative stress and antioxidant capacity to exercise in the overtrained state could be related to an inability to perform exercise effectively and impaired adaptation to exercise. 


\section{Introduction}

Repeated bouts of training coupled with sufficient recovery promotes adaptive responses that lead to improvements in performance. However, prolonged heavy training with incomplete recovery results in limited substrate availability, impaired protein synthesis and maladaptation (Radak et al., 2007). These conditions could lead to fatigue and overtraining syndrome (Steinacker et al., 2004; Meeusen et al., 2006a). The syndrome is characterized by an unanticipated reduction in performance, despite increased or maintained training challenges.

Several factors and symptoms have been linked to overtraining syndrome. However, there are no specific markers of impending overtraining and the exact pathophysiological chain of the syndrome is not known. Nonetheless, current theories on the role of cytokines, amino acids, muscle glycogen depletion, dysfunction of the hypothalamus and autonomic imbalance have been linked to the pathophysiology of overtraining syndrome (Meeusen et al., 2006b). Impaired antioxidant capacity and increased oxidative stress, where the production of reactive oxygen species overwhelms antioxidant defences, could be associated with chronic fatigue syndrome (Smirnova \& Pall, 2003), and might also be related to overtraining syndrome (Tiidus, 1998; Finaud et al., 2006). Animal (Ogonovszky et al., 2005; Zoppi \& Macedo, 2008) and human based studies have provided evidence that a progressively increased training volume can produce symptoms of overtraining (Vollaard et al., 2006; Margonis et al., 2007). Overload training can lead to an impaired antioxidant defence and a lack of anticipated adaptations to training (Smith, 2000), as well as a distortion of the redox balance (Finaud et al., 2006). Furthermore, 
overload training can induce inflammation, which is also related to oxidative stress. Extreme increases in training volume can lead to a substantial rise in inflammation and apoptosis markers (Fatouros et al., 2006). However, there is still no strong evidence to support any of these theories, so precise mechanisms responsible for overtraining syndrome remain unknown (Meeusen et al., 2006a).

The aim of the present study was to evaluate the association between oxidative stress and disrupted redox control, and overtraining syndrome. Oxidative stress markers and antioxidant capacity were studied in athletes diagnosed with severe overtraining syndrome and in control athletes before and after a six-month recovery period, both at rest and immediately after an exercise test to volitional exhaustion. It was hypothesised that overtrained athletes would exhibit increased oxidative stress and impaired antioxidant capacity compared with trained controls at baseline, and that an offset of this imbalance would occur after a six-month recovery period.

\section{Methods}

\section{Participants}

The participants for the present study were athletes who already had overtraining symptoms, as it is unacceptable to develop a study in which athletes are deliberately trained to develop overtraining syndrome. These athletes were recruited via a newspaper advertisement that covered the whole of Finland (Uusitalo et al., 2006). Seven overtrained (OT, two women and five men; six endurance athletes and one ice hockey player) and ten control athletes (five women and five men; nine endurance 
athletes and one sprinter) with no smoking history participated in the present study. Table 1 presents the baseline characteristics of the OT and control athletes. The OT athletes were heavier than the controls $(\mathrm{P}=0.037)$; otherwise the groups were similar in terms of their physical characteristics. The diagnostic criteria of overtraining syndrome were that the athlete: 1) had suffered from an unexplained decrement in physical performance and fatigue even after a recovery time of at least 3 weeks, which was verified by carefully interviewing the athletes and their coaches; 2) had been certified to be otherwise healthy based on a clinical examination, and several laboratory and physiological measurements performed at the Department of Clinical Physiology and Nuclear Medicine, Kuopio University Hospital, Finland (Uusitalo et al., 2004); 3) had a training history consistent with the indications of overtraining, i.e., the athletes had progressively increased their training volume and intensity for up to 6 months before overtraining symptoms appeared, and had continued training after this point without sufficient recovery time. Training logbooks of the previous months were examined to reveal the athletes' training histories. An additional criterion for overtraining syndrome was a slow recovery (not recovered after three weeks of rest or light training) of performance to normal, as well as a slow recovery of the symptoms related to overtraining.

All athletes recruited to the study fulfilled the criteria set for overtraining syndrome. In addition, the recovery for most of these athletes lasted more than 12 months, which is indicative of severe overtraining syndrome. Athletes participating in this study were part of a larger group of twelve OT athletes (6 males and 6 females). Our group has recently reported that these OT athletes were moderately depressed (Uusitalo et al., 2006), and presented with higher perceived stress than the controls 
(Hynynen et al., 2006). Moreover, these athletes had attenuated cardiac autonomic modulation during orthostatic tests and cognitive tasks (Hynynen et al., 2008) . In overtrained athletes, maximal oxygen uptake $\left(\mathrm{VO}_{2} \max \right)$ has been shown to decrease (Uusitalo et al., 1998b). However, in the present study $\mathrm{VO}_{2} \max$ results for OT athletes in healthy condition were not available. The tests were performed after the diagnosis of overtraining syndrome, and previous tests performed in the same laboratory for the comparison were not in use. In addition, comparisons between control and OT athletes had to be made with caution (Table 1 and Table 2), because of differences in sports events and the sex of participants between the groups, which might influence the results of maximal exercise tests. The study and control groups were similar (Table 1) concerning aerobic capacity, which is known to have an impact on markers of oxidative stress and antioxidant status (Jenkins et al., 1984; Rush \& Sandiford, 2003). All athletes were fully informed of the experimental protocol and the measurements, and provided written consent to participate in the study. The study protocol was approved by the ethics committee of Kuopio University Hospital, Kuopio, Finland.

\section{Experimental design}

Measurements were performed at baseline, i.e. at the time where OT athletes were suffering from severe overtraining syndrome, and after a six-month recovery period. Recovery for the OT group consisted of relative rest and an alternative form of exercise (involving a sport discipline that was different to the athlete's own competitive sport discipline) depending on the athlete's overall well-being. Training was initially limited to a maximum of 20 min of walking, three times a week. The 
volume of activity increased based on the athletes' own perceived abilities, gradually incorporating different activities (stretching, gymnastics, light speed or gym training). Blood samples were collected from an antecubital vein before and immediately after a cardiopulmonary exercise test to volitional exhaustion. The test consisted of a continuous, incremental cycle-ergometer (Ergoline Ergo-metrics 900, West-Germany) step test starting at $20 \mathrm{~W}$, and increasing by $20 \mathrm{~W}$ every minute until exhaustion. To define maximal aerobic capacity $\left(\mathrm{VO}_{2} \mathrm{max}\right)$, pulmonary gas exchange values were measured on-line using a Sensor Medix Vmax 29 (USA) breath-bybreath system, and mean values were calculated every 30 seconds for statistical analysis. The analyzer was calibrated with known volumes and gas concentrations before every test and verified immediately after. Heart rate was recorded on-line (using a Marquette Hellige Cardiosoft, Germany), and rating of perceived exertion (RPE) was assessed at the end of each stage. Standard physiological criteria were used to determine maximal effort (ASCM, 2001). Exercise time to exhaustion (EXtime; min: sec), maximal power output (Pmax; W) and maximal heart rate (HRmax; bpm) were all determined based on the volitional-exhaustion test. Technical error for Pmax and HRmax were 1-2 \% based on manufacturers' manuals. Repeatability of Pmax and HRmax in an exercise test to volitional exhaustion among athletes has reported to be $4 \%$ and 5\%, respectively (Bingisser et al., 1997).

The athletes were admitted to the laboratory at 8 a.m., two hours after a light breakfast. Before the measurements started, the OT athletes were interviewed and examined to ensure that they still fulfilled the overtraining syndrome criteria. The athletes used a self-reported scale to evaluate their positive and negative mood states during the previous two weeks. The four-point scale included six positive and nine 
negative items, and the means of both items were calculated. The reliability of the mood scale has been previously established (Uusitalo et al., 1998a): The Cronbach alpha coefficient for positive items is 0.82 and for negative items 0.83 . The athletes were instructed not to drink coffee, tea, chocolate or cola drinks on the morning of the measurements or the previous evening, and also not to drink alcohol within 48 hours of the measurements. They were also instructed to abstain from strenuous activity the day before testing. Data were collected over a three-year period because of the rare nature of severe overtraining syndrome. The women were measured at various stages of the menstrual cycle. Three of the control athletes and one OT athlete were users of oral contraceptives. At baseline, one OT athlete using oral contraceptives was measured at the end of the follicular phase, and one non-user in the middle of the menstrual cycle. Among the controls, two oral contraceptive users were measured at the beginning of the follicular phase, one non-user in the middle of the menstrual cycle and two in the luteal phase, one of whom was a user and one a non-user.

Biochemical analysis

Blood samples were collected into lithium-heparin tubes and centrifuged immediately after collection at $1200 \mathrm{~g}$ and $+4{ }^{\circ} \mathrm{C}$ for 15 minutes to separate the plasma. Plasma samples were stored in multiple portions at $-80^{\circ} \mathrm{C}$ until analysis. Samples were thawed only once for the measurements of oxidative stress and antioxidant markers. Exercise-induced relative changes in plasma were calculated using the equation: (after - before)/before $* 100$, and expressed as $\% \Delta$. Protein carbonyls, markers of protein oxidative damage, were measured using an ELISA 
method as previously described (Oksala et al., 2007). Oxygen radical absorbance capacity (ORAC) was used for the measurement of antioxidant capacity, which was performed using a multi-well plate reader according to methods described previously (Kinnunen et al., 2005). Nitrotyrosine concentrations were determined with the ELISA method using a commercial kit (HyCult biotechnology b.v; Netherlands). Total malondialdehyde in plasma was measured according to the method of GerardMonnier et al. (Goldfarb et al., 2005). As estimates of plasma volume change after exercise (Dill \& Costill, 1974) were small and similar for both groups, it was not deemed necessary to correct any plasma variables for changes in plasma volume. The intra-assay coefficients of variation for protein carbonyls, ORAC, nitrotyrosine and malondialdehyde assays were $5.9 \%, 8.1 \%, 10.0 \%$ and $6.2 \%$, respectively, and interassay coefficients of variation were $9.2 \%, 11.3 \%, 12.7$ and $10.6 \%$, respectively. Protein carbonyls and ORAC measurements were performed in triplicate.

\section{Statistical analysis}

Statistical analysis was performed using SPSS statistical software (SPSS, Inc., Chicago, IL, USA; version 12.0.1). The Shapiro-Wilks-test was used to test for normal distribution of variables. Normality assumptions for malondialdehyde and nitrotyrosine results could not be met, and data were log transformed before statistical analysis. An independent t-test was used to compare stature and mood states in each group at baseline. Nitrotyrosine results were not normalized after log transforming, so the nonparametric Kruskal-Wallis test was used to compare the differences between groups, and Friedman and Wilcoxon's pairwise tests were used to compare values in the groups. For the other variables a mixed-design factorial 
ANOVA [group (control vs. OT athletes) $\mathrm{x}$ exercise (before vs. after) $\mathrm{x}$ time (baseline vs. 6-month recovery)], with least significant difference (LSD) as the post hoc test, was used to identify differences between and within the groups. In addition, the effect of group, exercise and time and their interaction were calculated. Cohen's effect sizes $($ partial eta squared $=$ ES) $($ Cohen, 1988) and 95\% confidence intervals (CI) were also determined to estimate the meaningfulness of the changes/differences in means. Cohen (1988) classes an ES of 0.10 as a small effect, 0.25 a moderate effect, and 0.40 a large effect was used. If $95 \%$ confidence interval included zero, it was concluded that there was no significant effect, except for non-normally distributed nitrotyrosine. Pearson's correlation coefficients for normally distributed variables, and Spearman correlation coefficients for non-normally distributed variables, were computed to determine the relationship between oxidative stress markers and ORAC at baseline in the OT and control groups. During the follow-up, one female OT athlete discontinued the study and her data were excluded from analysis at the six-month time point. All values are reported as mean \pm standard deviation. Statistical significance was set to a p-value of 0.05 .

\section{Results}

During the experimental follow up, at the halfyear time point, one participant dropped out and the other OT athletes showed no signs of full recovery. Three of these athletes were not able to train regularly because of slow recovery from training and the presence of muscle and general fatigue, contributing to a detraining effect. Three athletes were able to train regularly with cautious planning, but their training 
volume had not returned to normal and they required more time for recovery from a training session than usual.

At baseline, the mean score of negative mood states was higher in OT than in controls (mean difference $0.7,95 \%$ CI 0.1 to $1.3, \mathrm{P}=0.009$, ES=0.45), indicating severe overtraining syndrome in the OT athletes (Uusitalo et al., 1998b). Positive mood states did not differ between groups (mean difference $-0.5,95 \%$ CI -1.0 to 0.1 , $\mathrm{P}=0.10$, $\mathrm{ES}=0.20$ ). The RPE was 20 at the end of exercise test to volitional exhaustion, both at baseline and after a six-month recovery period in both groups, and there were no differences in RPE at either time point between or within groups (Table 2). There was a group $\mathrm{x}$ time interaction for EXtime $(\mathrm{P}=0.021)$. After sixmonths of recovery, EXtime decreased in OT by $-6.8 \pm 7.9 \%$ (95\% CI -15.0 to $1.4 \%$, $\mathrm{P}=0.019 \mathrm{ES}=0.33)$ and $\mathrm{Pmax}$ by $-5.8 \pm 5.5 \%(95 \% \mathrm{CI}-0.1$ to $-11.6 \%, \mathrm{P}=0.028$, $\mathrm{ES}=0.30$ ). There were no other differences in EXtime, Pmax or HRmax at baseline or after the six-month recovery period between or within groups (Table 2). Body mass did not change after the six-month recovery period in either group.

\footnotetext{
$* * *$ Table 1 near here $* * *$

***Table 2 near here***
}

Oxidative stress markers

Resting protein carbonyls were higher in OT than in controls at baseline (mean difference $0.03 \mathrm{nmol} \cdot \mathrm{mg}^{-1}, 95 \%$ CI 0.01 to $0.05 \mathrm{nmol} \cdot \mathrm{mg}^{-1}, \mathrm{P}=0.003, \mathrm{ES}=0.40$ ) (Figure 1). Compared with the resting values, exercise to exhaustion caused an 
increase in protein carbonyls at baseline in controls (mean change $10.6 \pm 14.9 \%, 95 \%$ CI 0.1 to $21.3 \%, \mathrm{P}=0.017, \mathrm{ES}=0.33$ ), but not in OT (mean change $-1.0 \pm 5.6 \%, 95 \%$ CI -6.2 to $4.2 \%, \mathrm{P}=0.772$, $\mathrm{ES}=0.00$ ). After six months, exercise did not increase protein carbonyls in either group (controls mean change $7.5 \pm 15.6 \%, 95 \% \mathrm{CI}-3.7$ to $18.7 \%, \mathrm{P}=0.123, \mathrm{ES}=0.16$; OT mean change $6.1 \pm 14.8 \%, 95 \% \mathrm{CI}-9.4$ to $21.7 \%$, $\mathrm{P}=0.370, \mathrm{ES}=0.06)$. There was a main effect of exercise $(\mathrm{P}=0.0001)$ and group $\mathrm{x}$ exercise interaction $(\mathrm{P}=0.024)$ for malondialdehyde. In the controls at baseline, exercise caused an increase in malondialdehyde (mean change $27.5 \pm 23.8 \%, 95 \%$ CI 16.7 to $64.9 \%, \mathrm{P}=0.001, \mathrm{ES}=0.56$ ) and nitrotyrosine (mean change $10.3 \pm 18.1 \%, 95 \%$ CI -2.7 to $23.2 \%, \mathrm{P}=0.022$ [nonparametric test]), but after six months only malondialdehyde increased significantly (mean change $40.8 \pm 33.6 \%$, $95 \%$ CI 16.7 to 64.9\%, $\mathrm{P}=0.0001, \mathrm{ES}=0.64)$. In $\mathrm{OT}$ at baseline, exercise lead to an increase in nitrotyrosine (mean change $4.4 \pm 4.9 \%, 95 \%$ CI -0.2 to $8.9 \%, \quad \mathrm{P}=0.046$ [nonparametric test]), but not in malondialdehyde (mean change $13.6 \pm 15.2 \%, 95 \%$ CI -0.4 to $27.6 \%, \mathrm{P}=0.085$, ES=0.24). After the six month recovery, no exercise related changes were seen in either of these parameters in OT (Table 3). In contrast, OT had higher nitrotyrosine levels at the end of exercise after six months than at baseline (mean difference $14.0 \pm 18.1 \%, 95 \%$ CI -4.9 to $32.9 \%, \quad \mathrm{P}=0.046$ [nonparametric test]) (Table 3).

***Figure 1 near here $* * *$

***Figure 2 near here*** 
Antioxidant capacity

There was a main effect of exercise $(\mathrm{P}=0.006)$ and group $\mathrm{x}$ exercise interaction $(\mathrm{P}=0.037)$ also for ORAC. ORAC increased with exercise in the controls, both at baseline (mean change $13.3 \pm 11.1 \%, 95 \%$ CI 5.4 to $21.3 \%, \mathrm{P}=0.008, \mathrm{ES}=0.40$ ) and after six months (mean change $19.1 \pm 19.1 \%$, 95\% CI 5.4 to $32.7 \%, \mathrm{P}=0.003$, $\mathrm{ES}=0.47$ ), but not in OT (baseline mean change $5.7 \pm 15.9 \%, 95 \%$ CI -9.0 to $20.5 \%$, $\mathrm{P}=0.323, \mathrm{ES}=0.05 ;$ 6-month mean change $1.8 \pm 11.3 \%, 95 \% \mathrm{CI}-10.0$ to $13.7 \%$, $\mathrm{P}=0.928, \mathrm{ES}=0.00$ )(Figure 2). At baseline, only OT exhibited strong negative correlations $(\mathrm{r}=-0.98, \mathrm{P}=0.0001)$ between $\mathrm{ORAC}$ before and protein carbonyls after exercise; a lower ORAC before exercise was associated with higher protein carbonyls at the end of exercise (Table 4).

$* * *$ Table 3 near here $* * *$
$* * *$ Table 4 near here $* * *$

\section{Discussion}

To our knowledge, there are no previous reports that have investigated oxidative stress in athletes with overtraining syndrome, with the exception of a small number of studies where participants were trained intensively without any clinical diagnosis or symptoms of overtraining (Vollaard et al., 2006; Margonis et al., 2007). In the present study, overtraining status was associated with an increase in resting oxidative stress detected as higher protein carbonyl levels in OT athletes than controls. 
Furthermore, this study revealed that responses to acute exercise-induced stress were diminished in OT athletes.

The results demonstrating increased oxidative stress in overtraining state, even in resting conditions, are in agreement with a study by Margonis et al. (Margonis et al., 2007), where resting protein carbonyl levels increased following intense training, and peaked with progressively increased training volume. Although regular exercise training improves resistance to oxidative stress, decompensation and a lack of adaptation during overtraining may have the opposite effect. In accordance with the results of this study, Smirnova and Pall (2003) reported increased resting protein carbonyl levels in patients with chronic fatigue syndrome, which supports the hypothesis that increased oxidative stress is associated with overtraining syndrome. Differences between the OT and control groups in resting protein carbonyl levels may be attributed to phagocytic cell invasion of damaged muscle, which generates a substantial amount of reactive oxygen species, and is accompanied by underlying inflammation and soreness (Tiidus, 1998).

Unlike the changes in protein carbonyls (as a marker of protein oxidation), malondialdehyde (an indicator of lipid peroxidation) did not differ between groups at rest. Different mechanisms or factors may thus have influenced oxidative damage to lipids and proteins (Sen et al., 1997). Moreover, protein oxidation has been suggested to be of greater importance in cell toxicity than lipid peroxidation (Sandy et al., 1988). Although increased formation of nitrotyrosine is a marker of oxidative protein modification, there was no difference between groups in this study, which may be due to the wide variation of nitrotyrosine levels in the controls. In contrast to the 
protein carbonyl results, there was a trend for higher nitrotyrosine values at rest in controls than OT. Nitric oxide production may influence nitrotyrosine levels and increased nitrotyrosine content alone. Accordingly, nitrotyrosine may not be a sufficiently sensitive marker to assess training or acute exercise-induced changes in oxidative stress (Rush et al., 2003). Although the number of OT athletes did not differ from that in previous overtraining studies (Barron, 1985; Urhausen et al., 1998; Meeusen et al., 2004), the low number of participants probably accounts for the lack of statistical power and is a limitation in our study. The low number increases the likelihood of a type 2 error, and thus possible differences were not detected between control and OT athletes in resting malondialdehyde and nitrotyrosine levels.

Because of the difficulties associated with obtaining OT athletes, men and women athletes were recruited for this study. Furthermore, for practical reasons, female athletes were in different phases of the menstrual cycle at the time of data collection. There is evidence in the literature that estrogens have antioxidant properties (Joo et al., 2004; Vina et al., 2006), suggesting an influence of sex and menstrual-cycle status on oxidative stress and antioxidant capacity. Conversely, Chung et al. (1999) demonstrated that exercise-induced oxidative stress was independent of menstrual cycle. At baseline in the present study, three female controls were measured at the phase of the menstrual cycle where oestrogen levels are low, and thus oxidative stress could be high and antioxidant capacity weak. In spite of this, OT athletes exhibited increased oxidative stress at rest compared with controls. Excluding women from the study would have further decreased the power in the analysis. Comparisons of men OT athletes with men controls, revealed that the OT group still 
exhibited higher oxidative stress at rest than controls. Resting protein carbonyl levels and malondialdehyde was higher in $\mathrm{OT}(\mathrm{P}<0.05)$ than in controls.

Some exercise-induced oxidative stress is a physiological response, since at physiological concentrations, reactive oxygen species serve as messengers and regulate cellular adaptations (Pattwell \& Jackson, 2004). In the present study, responses to acute exercise-induced stress were diminished in OT; protein carbonyls and malondialdehyde did not increase as a result of exercise, and antioxidant protection could not react (ORAC was not induced). In contrast, malondialdehyde and ORAC responded to exercise in the controls, as observed in previous human studies (Schmidt et al., 2002; Fatouros et al., 2004). However, the OT athletes felt that their performance was restricted because of muscle fatigue, which may be associated with overtraining (Smith, 2000). Acute aerobic exercise to exhaustion resulted in a 16-fold increase in $\mathrm{VO}_{2}$ max above resting levels in OT, and RPE values in the tests were maximal in both groups, indicating overall maximal effort. Despite these indicators, the effort and exercise challenge might not have been strenuous enough to induce oxidative stress in these high-standard OT athletes.

In the present study, low antioxidant capacity at rest was associated with high exercise-induced protein oxidation and increased oxidative stress in OT, indicating poorer protection against exercise-induced protein oxidation. However, causality cannot be established here. Schmidt et al. (2002) reported that physically active male participants with low ORAC had increased oxidative stress markers after a 24-day training period. Alessio et al. (2000) reported increased ORAC after exhaustive exercise in healthy recreational athletes. Similarly, in this study there were increases 
in ORAC and protein carbonyl levels in response to acute exercise only in healthy control athletes and not in OT. Although ORAC values for the OT group prior to diagnosis of overtraining are not available, overtraining may have impaired total antioxidant capacity in OT (Margonis et al., 2007). This attenuated resting antioxidant capacity combined with lower exercise-induced oxidative stress in OT athletes may explain an abolished activation of antioxidant capacity in OT during exercise. An alternative explanation for impaired antioxidant responses to acute exercise-induced stress is that overtraining is a maladaptation state (Ogonovszky et al., 2005; Radak et al., 2007) and an organism's ability to cope with different tasks seems to be weakened (Barron, 1985; Urhausen et al., 1998; Meeusen et al., 2004; Hynynen et al., 2006, , 2008). However, a recovery period did not reverse this type of response, which could be a consequence of detraining (Fatouros et al., 2004).

Training during the six-month recovery period was evaluated by interviews and training diaries. During that time, OT participants were not able to train in their usual manner (i.e. before overtraining syndrome) because of severe fatigue and symptoms of overtraining. After the six-month period, Pmax and EXtime decreased and HRmax tended to decrease in OT compared to baseline values. These results could be a consequence of detraining rather than progressive overreach training, where a shortterm disturbance of homeostasis occurs so that further training adaptations can be attained (Hynynen et al., 2008). In addition, the higher nitrotyrosine levels at the end of exercise in OT after six-months compared with baseline can be explained by the detraining effect (Fatouros et al., 2004). In the only previous study where nitrotyrosine levels have been tracked during training and detraining in humans, training elicited a reduction in nitrotyrosine concentration after acute maximal 
exercise but not at rest (Fatouros et al., 2004). In that study, nitrotyrosine levels returned to pre-training levels after four months of detraining, and changes in nitrotyrosine after exercise seemed to be more sensitive to training and detraining effects than resting nitrotyrosine levels (Fatouros et al., 2004). The mechanism underlying the present findings that after six months of recovery, changes in nitrotyrosine occur only after exercise and not at rest, is still not fully understood.

In conclusion, antioxidant protection against exercise-induced acute stress was impaired and resting oxidative stress increased in OT athletes. In this study, protein carbonyls, malondialdehyde and nitrotyrosine concentrations were used as oxidative stress markers, among which protein carbonyls seem to be a promising diagnostic tool for early detection of overtraining. Our results suggest that increased oxidative stress plays a role in the pathophysiology of overtraining syndrome. Thus, monitoring physiological responses to acute exercise, including oxidative stress and antioxidant measures, could be useful additional tools to determine the need for adequate recovery in athletes. Further investigation of the pathophysiology of overtraining is still warranted to find better ways to detect overtraining syndrome in athletes.

\section{Acknowledgements}

The study was supported by grants from the Finnish Ministry of Education and the Medical Research Fund of Kuopio University Hospital (project number 5031318) and COST actions B35 and BM0602. 


\section{References}

ACSM, American College of Sports Medicine (2001). Guidelines for exercise testing and prescription. Baltimore, Lippincott Williams \& Wilkins.

Alessio, H. M., Hagerman, A. E., Fulkerson, B. K., Ambrose, J., Rice, R. E. \& Wiley, R. L. (2000). Generation of reactive oxygen species after exhaustive aerobic and isometric exercise. Medicine and Science in Sports and Exercise, 32, $1576-1581$.

Barron, J. F. (1985). Hypothalamic dysfunction in overtrained athletes. Journal of Clinical Endocrinology \& Metabolismb, 60, 803-807.

Bingisser, R., Kaplan, V., Scherer, T., Russi, E. W. \& Bloch, K. E. (1997). Effect of training on repeatability of cardiopulmonary exercise performance in normal men and women. Medicine and Science in Sports and Exercise, 29, 1499-1504.

Cohen, J. (1988). Statistical power analysis for the behavioral sciences, Hillsdale, NJ, Lawrence Erlbaum.

Dill, D. B. \& Costill, D. L. (1974). Calculation of percentage changes in volumes of blood, plasma, and red cells in dehydration. Journal of Applied Physiology, 37, 247-248.

Fatouros, I. G., Jamurtas, A. Z., Villiotou, V., Pouliopoulou, S., Fotinakis, P., Taxildaris, K. \& Deliconstantinos, G. (2004). Oxidative stress responses in older men during endurance training and detraining. Medicine and Science in Sports and Exercise, 36, 2065-2072. 
Fatouros, I. G., Destouni, A., Margonis, K., Jamurtas, A. Z., Vrettou, C., Kouretas, D., Mastorakos, G., Mitrakou, A., Taxildaris, K., Kanavakis, E. \& Papassotiriou, I. (2006). Cell-free plasma DNA as a novel marker of aseptic inflammation severity related to exercise overtraining. Clinical Chemistry, 52, 1820-1824.

Finaud, J., Lac, G. \& Filaire, E. (2006). Oxidative stress : relationship with exercise and training. Sports Medicine, 36, 327-358.

Goldfarb, A. H., Bloomer, R. J. \& McKenzie, M. J. (2005). Combined antioxidant treatment effects on blood oxidative stress after eccentric exercise. Medicine and Science in Sports and Exercise, 37, 234-239.

Hynynen, E., Uusitalo, A., Konttinen, N. \& Rusko, H. (2006). Heart rate variability during night sleep and after awakening in overtrained athletes. Medicine and Science in Sports and Exercise, 38, 313-317.

Hynynen, E., Uusitalo, A., Konttinen, N. \& Rusko, H. (2008). Cardiac autonomic responses to standing up and cognitive task in overtrained athletes. International Journal of Sports Medicine, 29, 552-558.

Jenkins, R. R., Friedland, R. \& Howald, H. (1984). The relationship of oxygen uptake to superoxide dismutase and catalase activity in human skeletal muscle. International Journal of Sports Medicine, 5, 11-14.

Joo, M. H., Maehata, E., Adachi, T., Ishida, A., Murai, F. \& Mesaki, N. (2004). The relationship between exercise-induced oxidative stress and the menstrual cycle. European Journal of Applied Physiology, 93, 82-86. 
Kinnunen, S., Hyyppa, S., Lehmuskero, A., Oksala, N., Maenpaa, P., Hanninen, O. \& Atalay, M. (2005). Oxygen radical absorbance capacity (ORAC) and exerciseinduced oxidative stress in trotters. European Journal of Applied Physiology, 95, $550-556$.

Margonis, K., Fatouros, I. G., Jamurtas, A. Z., Nikolaidis, M. G., Douroudos, I., Chatzinikolaou, A., Mitrakou, A., Mastorakos, G., Papassotiriou, I., Taxildaris, K. \& Kouretas, D. (2007). Oxidative stress biomarkers responses to physical overtraining: implications for diagnosis. Free Radical Biology and Medicine, 43, 901-910.

Meeusen, R., Piacentini, M. F., Busschaert, B., Buyse, L., De Schutter, G. \& StrayGundersen, J. (2004). Hormonal responses in athletes: the use of a two bout exercise protocol to detect subtle differences in (over)training status. European Journal of Applied Physiology, 91, 140-146.

Meeusen, R., Duclos, M., Gleeson, M., Rietjens, G., Steinacker, J. \& Urhausen, A. (2006a). Prevention, diagnosis and treatment of the Overtraining Syndrome. European Journal of Applied Physiology, 6, 1-14.

Meeusen, R., Duclos, M., Gleeson, M., Rietjens, G., Steinacker, J. \& Urhausen, A. (2006b). The Overtraining Syndrome - facts \& fiction. European Journal of Applied Physiology, 6, 263.

Ogonovszky, H., Sasvari, M., Dosek, A., Berkes, I., Kaneko, T., Tahara, S., Nakamoto, H., Goto, S. \& Radak, Z. (2005). The effects of moderate, strenuous, and overtraining on oxidative stress markers and DNA repair in rat liver. Canadian Journal of Applied Physiology, 30, 186-195. 
Oksala, N. K., Paimela, H., Alhava, E. \& Atalay, M. (2007). Heat shock preconditioning induces protein carbonylation and alters antioxidant protection in superficially injured guinea pig gastric mucosa in vitro. Digestive Diseases and Sciences, 52, 1897-1905.

Pattwell, D. \& Jackson, M. J. (2004). Contraction-induced oxidants as mediators of adaptation and damage in skeletal muscle. Exercise and Sport Science Reviews, $32,14-18$.

Radak, Z., Chung, H. Y., Koltai, E., Taylor, A. W. \& Goto, S. (2007). Exercise, oxidative stress and hormesis. Ageing Research Reviews, 6.

Rush, J. W. \& Sandiford, S. D. (2003). Plasma glutathione peroxidase in healthy young adults: influence of gender and physical activity. Clinical Biochemistry, 36, $345-351$.

Rush, J. W., Turk, J. R. \& Laughlin, M. H. (2003). Exercise training regulates SOD1 and oxidative stress in porcine aortic endothelium. American Journal of Physiology. Heart and Circulatory Physiology, 284, H1378-1387.

Sandy, M. S., Di Monte, D. \& Smith, M. T. (1988). Relationships between intracellular vitamin E, lipid peroxidation, and chemical toxicity in hepatocytes. Toxicology and Applied Pharmacology, 93, 288-297.

Schmidt, M. C., Askew, E. W., Roberts, D. E., Prior, R. L., Ensign, W. Y., Jr. \& Hesslink, R. E., Jr. (2002). Oxidative stress in humans training in a cold, moderate altitude environment and their response to a phytochemical antioxidant supplement. Wilderness and Environmental Medicine, 13, 94-105. 
Sen, C. K., Atalay, M., Agren, J., Laaksonen, D. E., Roy, S. \& Hanninen, O. (1997). Fish oil and vitamin E supplementation in oxidative stress at rest and after physical exercise. Journal of Applied Physiology, 83, 189-195.

Smirnova, I. V. \& Pall, M. L. (2003). Elevated levels of protein carbonyls in sera of chronic fatigue syndrome patients. Molecular and Cellular Biochemistry, 248, 9395.

Smith, L. L. (2000). Cytokine hypothesis of overtraining: a physiological adaptation to excessive stress? Medicine and Science in Sports and Exercise, 32, 317-331.

Steinacker, J. M., Lormes, W., Reissnecker, S. \& Liu, Y. (2004). New aspects of the hormone and cytokine response to training. European Journal of Applied Physiology, 91, 382-391.

Tiidus, P. M. (1998). Radical species in inflammation and overtraining. Canadian Journal of Physiology and Pharmacology, 76, 533-538.

Urhausen, A., Gabriel, H. H. \& Kindermann, W. (1998). Impaired pituitary hormonal response to exhaustive exercise in overtrained endurance athletes. Medicine and Science in Sports and Exercise, 30, 407-414.

Uusitalo, A. L., Uusitalo, A. J. \& Rusko, H. K. (1998a). Exhaustive endurance training for 6-9 weeks did not induce changes in intrinsic heart rate and cardiac autonomic modulation in female athletes. International Journal of Sports Medicine, 19, 532-540. 
Uusitalo, A. L., Vanninen, E., Valkonen-Korhonen, M. \& Kuikka, J. T. (2006). Brain serotonin reuptake did not change during one year in overtrained athletes. International Journal of Sports Medicine, 27, 702-708.

Uusitalo, A. L., Huttunen, P., Hanin, Y., Uusitalo, A. J. \& Rusko, H. K. (1998b). Hormonal responses to endurance training and overtraining in female athletes. Clinical Journal of Sport Medicine, 8, 178-186.

Uusitalo, A. L., Valkonen-Korhonen, M., Helenius, P., Vanninen, E., Bergstrom, K. A. \& Kuikka, J. T. (2004). Abnormal serotonin reuptake in an overtrained, insomnic and depressed team athlete. International Journal of Sports Medicine, $25,150-153$.

Vina, J., Borras, C., Gomez-Cabrera, M. C. \& Orr, W. C. (2006). Part of the series: from dietary antioxidants to regulators in cellular signalling and gene expression. Role of reactive oxygen species and (phyto)oestrogens in the modulation of adaptive response to stress. Free Radical Research, 40, 111-119.

Vollaard, N. B., Cooper, C. E. \& Shearman, J. P. (2006). Exercise-induced oxidative stress in overload training and tapering. Medicine and Science in Sports and Exercise, 38, 1335-1341.

Zoppi, C. C. \& Macedo, D. V. (2008). Overreaching-induced oxidative stress, enhanced HSP72 expression, antioxidant and oxidative enzymes downregulation. Scandinavian Journal of Medicine and Science in Sports, 18, 67-76. 


\section{Figure Legends}

FIGURE 1. Protein oxidation measured as protein carbonyl levels before and immediately after an exercise test to volitional exhaustion at baseline and after the 6month recovery period in overtrained and control athletes. \#\# Difference between controls and overtrained athletes $(\mathrm{P}=0.003)$. * Significant increase due to exercise $(\mathrm{P}=0.017)$.

FIGURE 2. ORAC before and immediately after an exercise test to volitional exhaustion at baseline and after the 6-month recovery period in overtrained and control athletes.

** Significant increase due to exercise $(\mathrm{P}<0.01)$. There was a significant main effect of exercise $(\mathrm{P}=0.006)$ and group $\mathrm{x}$ exercise interaction $(\mathrm{P}=0.037)$. 
TABLE 1. Baseline characteristics of controls $(n=10)$ and overtrained athletes $(n=7)$.

\begin{tabular}{|c|c|c|c|c|}
\hline & \multicolumn{2}{|c|}{ Controls } & \multicolumn{2}{|c|}{ Overtrained } \\
\hline & Mean \pm SD & Range & Mean \pm SD & Range \\
\hline Age (yr) & $27 \pm 6$ & $20-39$ & $26 \pm 8$ & $16-39$ \\
\hline Body mass (kg) & $65 \pm 7$ & $51-78$ & $72 \pm 10 \#$ & $56-86$ \\
\hline Stature $(\mathrm{cm})$ & $171 \pm 7$ & $155-178$ & $176 \pm 9$ & $162-190$ \\
\hline BMI $\left(\mathrm{kg} \cdot \mathrm{m}^{-2}\right)$ & $22.1 \pm 1.6$ & $20.5-25.2$ & $22.8 \pm 1.6$ & $20.6-24.7$ \\
\hline Training (yr) & $8 \pm 5$ & $3-17$ & $9 \pm 5$ & $2-14$ \\
\hline $\mathrm{VO}_{2} \max \left(1 \cdot \min ^{-1}\right)$ & $3.7 \pm 0.7$ & $2.9-4.6$ & $3.8 \pm 0.8$ & $2.6-5.1$ \\
\hline $\mathrm{VO}_{2} \max \left(\mathrm{ml} \cdot \mathrm{kg}^{-1} \cdot \mathrm{min}^{-1}\right)$ & $57 \pm 8$ & $44-69$ & $53 \pm 8$ & $45-64$ \\
\hline
\end{tabular}


TABLE 2. Cycle-ergometer test results at baseline and after six months of recovery in controls and overtrained athletes. Pmax: maximal power output during exercise; EXtime: time to exhaustion; HRmax: maximum heart rate

\section{Controls}

\section{Overtrained}

Baseline 6-month 6-month

$\begin{array}{lcccccccccc}\text { Pmax (W) } & 288 & \pm & 63 & 288 & \pm 65 & 323 & \pm 66 & 303 & \pm 56 \# \\ \text { EXtime (min:sec) } & 14: 42 & \pm & 3: 10 & 14: 57 & \pm 3: 02 & 16: 25 & \pm 3: 14 & 15: 25 & \pm & 3: 53 \mathrm{a} \\ \text { HRmax (bpm) } & 192 & \pm & 6 & 192 & \pm 8 & 190 & \pm 11 & 185 & \pm 15 \\ \text { RPE } & 19.8 & \pm & 0.4 & 19.8 & \pm 0.3 & 20.0 & \pm & 0.0 & 20.0 & \pm 0.0\end{array}$

Values are means \pm SD. Different from baseline; \# $\mathrm{P}=0.019, \propto \mathrm{a}=0.028$. ${ }^{\mathrm{a}}$ Interaction, group $\mathrm{x}$ time, $\mathrm{P}=0.021$. 
TABLE 3. Plasma nitrotyrosine and malondialdehyde at baseline and after the 6-month recovery before and immediately after an exercise test to volitional exhaustion in controls and overtrained athletes.

Controls

Overtrained

\begin{tabular}{lllll}
\multicolumn{2}{c}{ Baseline } & \multicolumn{2}{c}{ 6-month } & \multicolumn{2}{c}{ 6-month } \\
\hline Before After & Before After & Before After & Before After
\end{tabular}

Nitrotyrosine

$(\mu \mathrm{mol})$

$1729 \quad 1842 * \quad 1664 \quad 1775 \quad 799$

823\# 789

$911 \not$

$\pm \mathrm{SD}$

1665

1718

1657

1666

1105

1112978

1186

Malondialdehyde

$\begin{array}{lllllllll}\left(\mathrm{nmol} \cdot \mathrm{ml}^{-1}\right)^{\text {aaa, a }} & 1.10 & 1.35^{* * *} & 0.93 & 1.33 * * * & 1.29 & 1.45 & 1.10 & 1.17 \\ \pm \mathrm{SD} & 0.50 & 0.57 & 0.24 & 0.53 & 0.47 & 0.39 & 0.25 & 0.42\end{array}$

Values are means $\pm \mathrm{SD}$. Different from before: $* * * \mathrm{P}<0.001, * \mathrm{P}=0.022, \# \mathrm{P}=0.046$. Different from baseline; $x \mathrm{P}=0.046 .{ }^{\text {aaa }}$ Main effect of exercise, $\mathrm{P}=0.0001$. $^{\mathrm{a}}$ Interaction, group x exercise, $\mathrm{P}=0.024$. 
TABLE 4. Correlation coefficients between oxidative stress markers and ORAC at baseline in controls and overtrained athletes.

\section{CONTROL}

ORAC
OVERTRAINED

\section{ORAC}

\begin{tabular}{lcccc} 
& Before & After & Before & After \\
\hline Before & -0.35 & 0.16 & -0.32 & 0.01 \\
After & -0.21 & 0.10 & $-0.98^{* * *}$ & -0.16 \\
Before & -0.18 & 0.25 & 0.14 & -0.04 \\
After & -0.18 & 0.13 & 0.29 & -0.16
\end{tabular}

Malondialdehyde $\left(\mathrm{nmol} \cdot \mathrm{ml}^{-1}\right)$

$-0.17$

$-0.34$

$-0.27$

0.23

After

$-0.25$

$-0.18$

$-0.16$

0.07

Before $=$ before an exercise test to volitional exhaustion. After = immediately after an exercise test to volitional exhaustion. $* * * \mathrm{P}=0.0001$. 


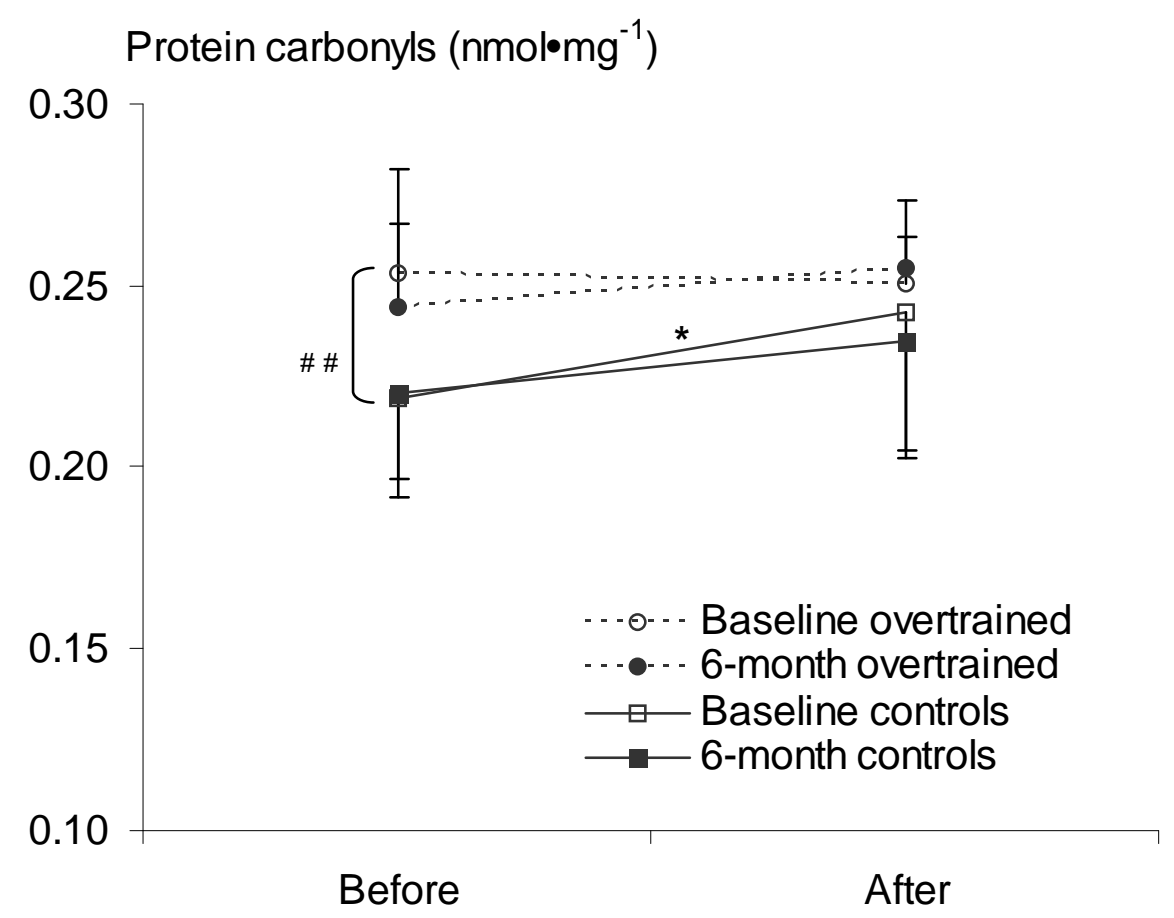


ORAC ( $\mu \mathrm{M}$ Trolox equivalents)

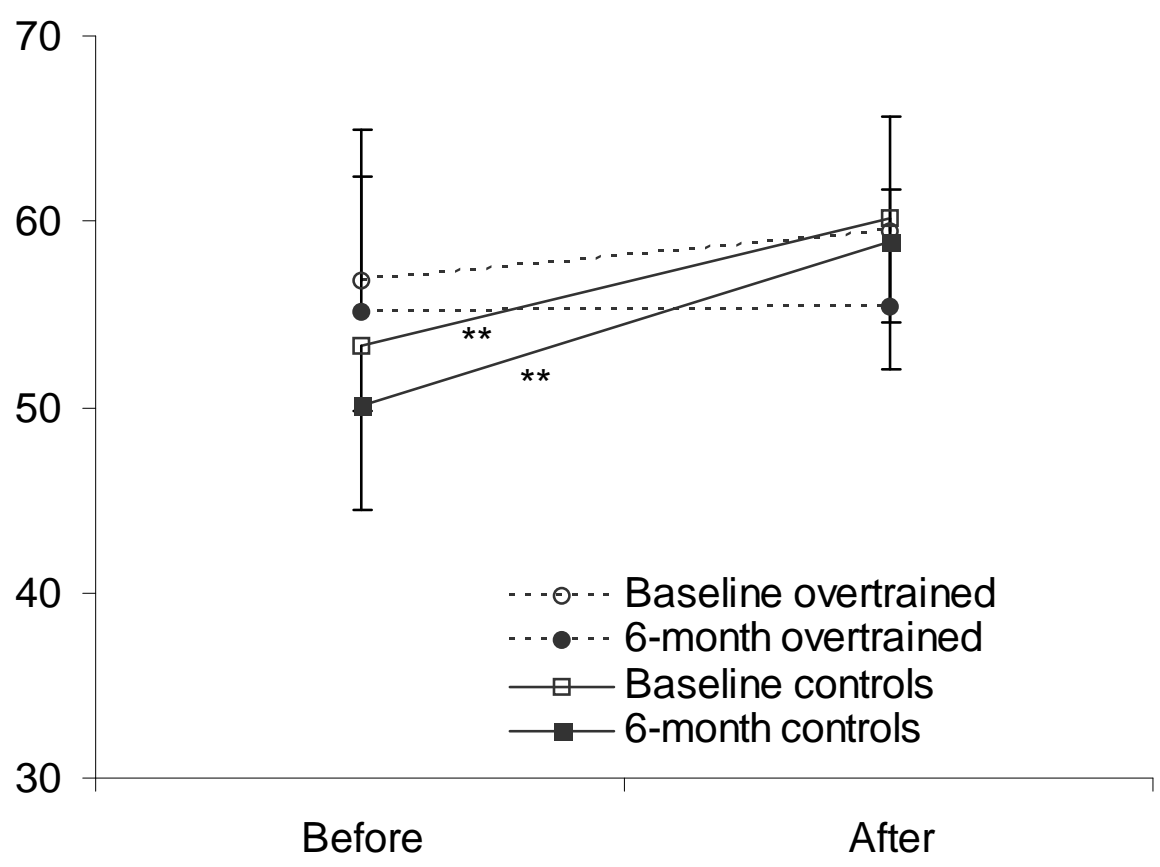

